\title{
Abstract: Assessment of Segmentation Dependence in Macroscopic Lung Cavity Extraction
}

\author{
Asmaa Khdeir ${ }^{1,2, *}$, Tobias Geimer ${ }^{1,2,3, *}$, Shuqing Chen ${ }^{1}$, Eric Goppert ${ }^{1}$, \\ Maximilian Dankbar ${ }^{1}$, Christoph Bert ${ }^{2,3}$, Andreas Maier ${ }^{1,3}$ \\ ${ }^{1}$ Pattern Recognition Lab, Friedrich-Alexander-Universität Erlangen-Nürnberg \\ ${ }^{2}$ Department of Radiation Oncology, Universitätsklinikum Erlangen, FAU Er-N \\ ${ }^{3}$ Erlangen Graduate School in Advanced Optical Technologies, FAU Er-N \\ *Both authors contributed equally. \\ asmaa.k.khdeir@fau.de
}

Training of respiratory motion models and population-based patient phantoms of the lung often requires the definition of the entire lung cavity region in the 4D-CT. To ease the workload of clinical experts, automatic selection is highly desirable. Many lung cavity extraction methods rely on a pre-segmented lung volume. We propose a simple yet fully automatic pipeline that preserves the $3 \mathrm{D}$ shape of the lung while also incorporating the chest wall and diaphragm, both of which carry significant respiratory information.

The proposed pipeline consists of three main parts: First, a convex-hull algorithm provides a mesh representation encompassing the lung region in continuous world coordinates. Second, raycasting w.r.t. the original dimensions returns a voxelized binary volume, and third, morphological operation includes the desired parts of the diaphragm and chest wall.

For seven 3D-CT patient datasets in end-inhale respiratory state, we evaluated the pipelineś robustness against the chosen segmentation method by comparing three different initial segmentations: 1) An intensity-based method using adaptive thresholding, 2) a registration-based atlas segmentation, and 3) segmentation by a 3D fully convolutional neural network.

A dice-score increase of up to 0.167 and average pair-wise sensitivity of $91.2 \%$ and specificity of $98.7 \%$ show that the pipeline is reasonably robust against varying initial segmentations, indicating that a simple intensity-based method provides similar results to complex deep-learning approaches. The resulting lung cavity can find use in population models of internal organ structures or serve as a cropping mask for the ground truth of motion models. 